\title{
A CONSTRUCTION FOR FITTING-SCHUNCK CLASSES
}

\author{
JOHN COSSEY and ELIZABETH A. ORMEROD
}

(Received 6 June 1986)

Communicated by $\mathrm{H}$. Lausch

\begin{abstract}
A method for constructing Fitting-Schunck classes is given: the method is an adaptation of one given by C. L. Kanes for constructing Fitting formations, and generalizes the Fitting-Schunck class construction given by Cossey in 1981. A criterion for deciding which of the Fitting-Schunck classes so constructed are formations is given.
\end{abstract}

1980 Mathematics subject classification (Amer. Math. Soc.): 20 F 16.

In [2], the first author constructed examples of Fitting classes that were Schunck classes, but not formations. The construction in that paper was based on an idea used by Trevor Hawkes in [4] to construct examples of non-saturated Fitting formations. This idea of Hawkes was generalized by Clive Kanes in his PhD thesis [8] to give a general method for constructing Fitting formations (an exposition of the method can be found in [3]). The purpose of this note is to show that the construction of Fitting-Schunck classes can be generalized in the same way, and to establish which of the classes so constructed are not formations. All groups in the remainder of this note are finite and soluble.

We recall the definition of a Fitting family given in [3]. Let $K$ be a field of prime characteristic $q$. Suppose that for each group $G$ we are given a class of modules $M(G) \subseteq \operatorname{Irr}_{K}(G)$ : let $\mathscr{M}$ be the family of all $M(G)$. Then we call $\mathscr{M}$ a Fitting family if it satisfies the following closure properties.

M1. The trivial irreducible $K G$-module is in $M(G)$.

M2. If $V \in M(G), N \triangleleft G$, with $N \subseteq C_{G}(V)$, then $V$ (regarded in the natural way as $G / N$-module) is in $M(G / N)$.

M3. If $V \in M(H)$, and $\varphi: G \rightarrow H$ is an epimorphism, then $V$ (regarded in the natural way as a $G$-module) is in $M(G)$.

(C) 1987 Australian Mathematical Society $0263-6115 / 87 \$ A 2.00+0.00$ 
M4. If $V \in M(G), N \triangleleft G$, and $U$ an irreducible constituent of $V_{N}$, then $U \in M(N)$.

M5. If $V \in \operatorname{Irr}_{K}(G), G=N_{1} N_{2}, N_{1}, N_{2}$ normal subgroups of $G$, and if for each irreducible constituent $U$ of $V_{N_{i}}$, we have $U \in M\left(N_{i}\right), i=1,2$, then $U \in M(G)$.

If $H / L$ is a $q$-chief factor of $G$, we can regard $H / L$ as a $G F(q) G$-module (by conjugation). We put $(H / L)^{K}=(H / L) \otimes_{G F(q)} K$ and then define classes $\Delta_{K}(G)$ and $\Gamma_{K}(G)$ of $K G$-modules by

$\Delta_{K}(G)=\left\{U \in \operatorname{Irr}_{K}(G): U\right.$ is isomorphic to a composition factor of $(H / L)^{K}$, for $H / L$ a complemented $q$ chief factor of $G\}$,

$\Gamma_{K}(G)=\left\{U \in \operatorname{Irr}_{K}(G): U\right.$ is isomorphic to a composition factor of $(H / L)^{K}$, $H / L$ a $q$ chief factor of $G\}$.

If now $\mathscr{M}$ is a Fitting family, we define a class $\mathbf{C}$ by setting

$$
\mathrm{C}=\left\{G: \Delta_{K}(G) \subseteq M(G), M(G) \in \mathscr{M}\right\} .
$$

That $\mathbf{C}$ is a Schunck class is immediate from its definition. We will show that it is a Fitting class.

The proof that $\mathbf{C}$ is normal product closed follows the proof of the corresponding fact in [2], using the properties of modules in $M(G)$ in place of the arguments of Hawkes; we omit the details.

For subnormal subgroup closure, we will show by induction on $|G|$ that if $G \in \mathrm{C}, N$ a maximal normal subgroup of $G$, and $H / L$ is a complemented $q$ chief factor of $N$, then the composition factors of $(H / L)^{K}$ lies in $M(G)$. If $M \leqslant N$ is a normal subgroup of $G$ contained in a complement in $N$ for $H / L$, then $M \leqslant C_{G}(H / L)$, and the result follows by induction and property $\mathbf{M} 2$. Since $O_{q^{\prime}}(N)$ is contained in any complement for a complemented $q$ chief factor of $N$, we may assume that $O_{q^{\prime}}(N)=1$. Moreover, by Theorem 1 of Barnes [1] we may assume that $H / L$ has been chosen so that $H \leqslant F^{n}(N)$ for some $n$ (where $F^{n}(N)$ is the $n$th term of the upper nilpotent series of $N$ ), and hence we may assume that $H \leqslant F(N)$, and $\Phi(N)=1$. Put $F=F(N)$ : then $F$ is completely reducible as $N$-module, and we may as well assume that $L=1$.

Let $U$ be the sum of all the irreducible submodules of $F$ isomorphic to a $G$-conjugate of $H$. Since $F$ is completely reducible, we have $F=U \oplus V$, where $V$ is the sum of all the irreducible submodules of $F$ not isomorphic to a $G$-conjugate of $H$. Moreover, $U, V$ are normal subgroups of $G$, and if $C$ is a complement of $H$ in $N, C \cap F$ will be a complement for $H$ in $F$, and so must contain $V$. Thus we may suppose that $V=1$.

Now let $Q / F=O_{q^{\prime}}(N / F)$, and let $S$ be a complement for $F$ in $Q$. Since $S$ is faithfully represented on $F$ and each irreducible submodule of $F$ (as $N$-module) is $G$-conjugate, all irreducible submodules of $F_{Q}$ are $G$-conjugate: none can be trivial, since $Q \triangleleft G$, and so $[F, Q]=[F, S]=F$. It follows that $Q=F S$ is normal 
in $G$, but $E S$ is not normal in $G$ for any normal subgroup $E$ of $G$ with $E$ properly contained in $F$. But then Theorem 1 of Higman [5] gives us that $F$ is complemented in $G$.

Since $N$ is a maximal normal subgroup of $G, G / N$ has prime order, $r$ say. Suppose first that $r \neq q$ : then $F$ is completely reducible as $G$-module by [7] Theorem 7.7.20, and it is now an easy check to see that every chief factor of $G$ contained in $F$ is complemented in $G$. Thus the composition factors of $H^{K}$ are in $M(N)$ by property M4.

Thus suppose that $r=q$. We have that $F$, as an $N$-module, is a direct sum of irreducibles, each isomorphic to a $G$-conjugate of $H$ : we claim that all composition factors of $F$, as $G$-modules, are isomorphic. Since $F$, as a $G$-module, is isomorphic to a quotient of $F_{N}^{G}$, it will be enough to show that all composition factors of $F_{N}{ }^{G}$ are isomorphic. If $W$ is isomorphic to a $G$-conjugate of $H$, then $W^{G}$ is isomorphic to $H^{G}$, and so it will be enough to show that all composition factors of $H^{G}$ are isomorphic. If $H^{G}$ is irreducible, there is nothing to prove, and so we assume that $H^{G}$ is reducible. It follows that $H$ is $G$-invariant, and then by the Mackey Subgroup Theorem and [7] Lemma 7.9.19 that $U_{N} \simeq H$ for each composition factor $U$ of $H^{G}$. But now, by extending to the algebraic closure of $G F(q)$ and using Corollary 7.9.13 of [7], we see that there is a unique $G$-module $W$ such that $W_{N} \approx H$. Thus we have all the composition factors of $H^{G}$ isomorphic, as required.

If $E$ is a maximal submodule of $F$, then $F / E$ is a complemented chief factor of $G$, and $(F / E)_{N} \simeq H$ : that the composition factors of $H^{K}$ lie in $M(N)$ now follows from property M4. This completes the proof.

Known Fitting families, such as those given in Section 4 of [3], can now be used to give examples of Fitting-Schunck classes. The example given in [2] is just the intersection of the class of $q$-nilpotent groups with the class given by using the Fitting family $\mathbf{M}_{q}^{q}(\mathbf{E}, \mathbf{S})$ (in the notation of [3]).

We want to be able to decide which of the classes we have constructed are formations. We are able to give a simple criterion: if $\mathscr{M}$ is a Fitting family, and $\mathbf{C}$ the corresponding class, then $\mathbf{C}$ is a formation if and only if for all $G$ and all $U, V \in M(G)$, every composition factor of $U \otimes V$ is in $M(G)$.

To establish this, we first suppose that $\mathbf{C}$ is a formation. Let $G \in \mathbf{C}$, and $U_{0}, V_{0} \in M(G)$. Let $U$ and $V$ be $G F(q) G$-modules such that $U^{K}$ and $V^{K}$ have composition factors isomorphic to $U_{0}$ and $V_{0}$ respectively. Put $H=(U \oplus V$, $U \otimes V$ ), the group constructed as in Hilfssatz 6.7.22 of Huppert [6], and then put $G_{0}=H G$. Then we have $U \otimes V \leqslant H^{\prime}$, and so $U \otimes V \leqslant \Phi(H) \leqslant \Phi\left(G_{0}\right)$. Thus $\Delta_{K}\left(G_{0}\right) \subseteq M\left(G_{0}\right)$ by property $\mathrm{M} 3$, and so $G_{0} \in \mathbf{C}$.

Now let $W$ be a composition factor of $U_{0} \otimes V_{0}$, and let $S / T$ be a composition factor of $U \otimes V$ with $W$ isomorphic to a composition factor of $(S / T)^{K}$. It will be enough to show that $W \in M\left(G_{0}\right)$; we then have that $H$ centralizes $W$, and so by 
properties $\mathrm{M} 2$ and $\mathrm{M} 1, W \in M(G)$. Since $W \in M\left(G_{0}\right)$ if and only if $W \in$ $M\left(G_{0} / T\right)$ (by properties $\mathrm{M} 2$ and M3), we consider $S_{1}=S / T, G_{1}=G_{0} / T$.

Let $L$ be the semidirect product of $S_{1}$ by $G_{1}$ : then $L$ is a subdirect product of two copies of $G_{1}$ and so is in C. But $S_{1}$ is a complemented chief factor of $L$, and so $W \in M(L)$. It then follows from properties M1 and M2 that $W \in M\left(G_{1}\right)$, as required.

In the other direction, to show that $\mathbf{C}$ is a formation we need to show that $\mathbf{C}$ is subdirect product closed. To do this, it will be enough to show that $\Gamma_{K}(G) \subseteq M(G)$ for all $G \in \mathbf{C}$. For then if $L$ is a subdirect product of $G, H \in \mathbf{C}$, we have that each module $U$ in $\Gamma_{K}(L)$ is also in $\Gamma_{K}(G)$ or $\Gamma_{K}(H)$ and so in $M(G)$ or $M(H)$. It then follows from properties M2 and M3 that $\Delta_{K}(L) \subseteq \Gamma_{K}(L) \subseteq M(L)$, and so $L \in \mathbf{C}$.

Put $W=O_{q^{\prime} q}(G) / Q$, where $Q / O_{q^{\prime}}(G)=\left(O_{q^{\prime} q}(G) / O_{q^{\prime}}(G)\right)$ : then $W^{K}$ is a direct sum of modules in $M(G)$ and so composition factors of tensor powers of $W^{K}$ are in $M(G)$ by assumption. Since $W$ is a faithful $G / O_{q^{\prime} q}(G)$-module, each irreducible $K\left(G / O_{q^{\prime} q}(G)\right)$-module is isomorphic to a composition factor of a tensor power of $W^{K}$ ([6] Satz 6.7.19), and so $M(G)$ contains all irreducible $K\left(G / O_{q^{\prime} q}(G)\right)$-modules. But if $H / L$ is a $q$-chief factor of $G$, we can regard $H / L$ as a $G / O_{q^{\prime} q}(G)$-module. It follows that the irreducible constituents of $(H / L)^{K}$ are in $M\left(G / O_{q^{\prime} q}(G)\right)$, and then by property $M 3$ in $M(G)$. Thus $\Gamma_{K}(G) \subseteq M(G)$, as required.

\section{References}

[1] D. W. Barnes, 'On complemented chief factors of finite soluble groups', Bull. Austral. Math. Soc. 7 (1972), 101-104.

[2] John Cossey, 'On Fitting classes that are Schunck classes', J. A ustral. Math. Soc. 30 (1981), 381-384.

[3] John Cossey and C. L. Kanes, 'A construction for Fitting formations', J. Algebra, to appear.

[4] T. O. Hawkes, 'On Fitting formations', Math. Z. 117 (1970), 177-182.

[5] Graham Higman, 'Complementation of abelian normal subgroups', Publ. Math. Debrecen 4 (1956), 455-458.

[6] B. Huppert, 'Endliche Gruppen I' (Die Grundlehren der Mathematischen Wissenschaften 137, Springer Verlag, Berlin, Heidelberg, New York, 1967).

[7] B. Huppert and N. Blackburn, Finite Groups II (Die Grundlehren der Mathematischen Wissenschaften 242, Springer Verlag, Berlin, Heidelberg, New York, 1982).

[8] C. L. Kanes, 'Constructions for Fitting formations'. (PhD Thesis, ANU, 1982).

Department of Mathematics, Faculty of Science

Australian National University

P. O. Box 4, Canberra, ACT

Australia 